\title{
Terbium ion-coordinated carbon dots for fluorescent aptasensing of adenosine 5'-triphosphate with unmodified gold nanoparticles
}

Mingdi $\mathrm{Xu}^{\mathrm{a}}$, Zhuangqiang Gao ${ }^{\mathrm{a}}$, Qian Zhou ${ }^{\mathrm{a}}$, Youxiu Lin ${ }^{\mathrm{a}}$, Minghua Lu ${ }^{\mathrm{b}, *}$, and Dianping Tang ${ }^{\mathrm{a}, *}$

${ }^{\text {a }}$ Key Laboratory of Analysis and Detection for Food Safety (MOE \& Fujian Province), Institute of Nanomedicine and Nanobiosensing, Department of Chemistry, Fuzhou University, Fuzhou 350108, PR China

${ }^{\mathrm{b}}$ Institute of Environmental and Analytical Science, School of Chemistry and Chemical Engineering, Henan University, Kaifeng 475004, Henan, PR China

\section{CORRESPONDING AUTHOR INFORMATION}

Phone: +86-591-2286 6125; fax: +86-591-2286 6135; e-mail: dianping.tang@ fzu.edu.cn (D. Tang) 


\section{ABSTRACT}

This work reports on a novel time-resolved fluorescent aptasensing platform for the quantitative monitoring of adenosine 5'-triphosphate (ATP) by interaction of dispersive/agglomerate gold nanoparticles (AuNPs) with terbium ion-coordinated carbon dots (Tb-CDs). To construct such a fluorescent nanoprobe, Tb-CDs with high-efficient fluorescent intensity are first synthesized by the microwave method with terbium ions $\left(\mathrm{Tb}^{3+}\right)$. The aptasensing system consists of ATP aptamer, AuNP and Tb-CD. The dispersive/agglomerate gold nanoparticles are acquired through the reaction of the aptamer with target ATP. Upon target ATP introduction, the aptamers bind with the analytes to form new aptamer-ATP complexes and coat on the surface of AuNPs to inhibit their aggregation in the high salt solution. In this case, the fluorescent signal of Tb-CDs is quenched by the dispersive AuNPs on the basis of the fluorescence resonance energy transfer (FRET). At the absence of target analyte, gold nanoparticles tend to aggregate in the high salt state even if the aptamers are present. Thus, the added Tb-CDs maintain their intrinsic fluorescent intensity. Experimental results indicated that the aptasensing system exhibited good fluorescent responses toward ATP in the dynamic range from $40 \mathrm{nM}$ to $4.0 \mu \mathrm{M}$ with a detection limit of $8.5 \mathrm{nM}$ at $3 s_{\text {blank }}$ criterion. The repeatability and intermediate precision is less than $9.5 \%$ at three concentrations including $0.04,0.4$ and $2.0 \mu \mathrm{M}$ ATP. The selectivity was acceptable toward guanosine 5'-triphosphate, uridine 5'-triphosphate and cytidine 5'-triphosphate. The methodology was applied to evaluate the blank human serum spiked with target ATP, and the recoveries (at 3 concentration levels) ranged between $97.0 \%$ and $103.7 \%$. Importantly, this detection scheme is rapid, simple, cost-effective, and does not require extensive sample preparation or separation.

KEYWORDS: fluorescent aptasensor, terbium ion-coordinated carbon dots, gold nanoparticles, fluorescence resonance energy transfer, adenosine 5'-triphosphate 


\section{Introduction}

Fluorescent nanomaterials have drawn great attention because of their potential applications in different research fields including bioimaging, optoelectronic devices and (bio-)sensings (Stanisavljevic et al., 2015; Wen et al., 2015). Previous reports have shown that the electron-accepting and transport properties of carbon nanomaterials could provide a convenient way to separate photogenerated electrons (Zhang et al., 2011a; Zhang et al., 2011b). Carbon dots (CDs), as small carbon nanomaterials with a size of below $10 \mathrm{~nm}$, have become one of the most promising nanostructures owing to their favorable photostability, low toxicity, flexible modification, biocompatibility, high water solubility, high photoluminescence quantum yields and excitation-dependent emissions (Schwenke et al., 2015; Wolfbeis, 2015). Wang et al. reported a simple one-step hydrothermal approach for green preparation of carbon dots with papaya as carbon source for effective fluorescent sensing of iron(III) and Escherichia coli (Wang et al., 2016a). Fong et al. developed a unique "turn on" fluorescence signaling strategy for highly specific detection of ascorbic acid using carbon dots as sensing probe (Fong et al., 2016). Nevertheless, one disadvantage of using carbon dots alone lies in easy oxidization, thus limiting in their practical application (Amjadi et al., 2015; Zhang et al., 2014; Chen et al., 2016). Therefore, exploring new nanomaterials based on carbon dots would be advantageous for the development of fluorescent nanoprobes.

Nanocomposites, as a multiphase solid material, have broadened significantly to encompass a large variety of systems such as one/two/three-dimensional and amorphous materials, made of distinctly dissimilar components and mixed at the nanometer size (Sabherwal et al., 2016; Prateek et al., 2016). Shi et al. synthesized nitrogen and phosphorous Co-doped carbon dots for fluorescent sensing of iron(III) in human serum and living cells (Shi et al., 2016). Zhang et al. prepared gold-carbon dots with unique properties for ratiometric fluorescence cellular imaging (Zhang et al., 2016). LeCroy et al. reviewed the functionalized carbon dots in the application of bioimaging and theranostics (LeCroy et al., 2016). Terbium, a silvery-white rare metal, is relatively stable in air as compared to other lanthanides. Terbium(III) cation possesses brilliantly fluorescent in a bright lemon-yellow color as a result of strong green emission line in combination with other lines in the organ and red. However, terbium like carbon dots easily oxidizes, and is 
thus used in its elemental or complex form for research (https://en.wikipedia.org/wiki/Terbium). Chen et al. synthesized terbium-doped carbon dots for highly selective fluorescence detection of 2,4,6-trinitrophenol (Chen et al., 2016). Tang et al. constructed a ratiometric fluorescent nanoprobe based on terbium functionalized carbon dots for the detection of an anthrax biomarker (Chen et al., 2015). Dong et al. further found that energy transfer from carbon dots to the rare-earth metal caused line-type $\mathrm{Tb}^{3+}$ emission with quantum yields up to $85 \%$ upon modification with terbium ions (Dong et al., 2014). Hence, terbium ion-coordinated carbon dots can be employed as the nanoprobes for fabrication of fluorescent sensing platform.

Aptamers, selected from random-sequence nucleic acid libraries by exponential enrichment (SELEX) process, are functional nucleic acids with highly specific recognition capabilities toward different targets including small molecules, metal ions and proteins (Crivian-Gaita and Thompson, 2016; Strehlitz et al., 2012). Importantly, the aptamers undergo the conformational changes once target binding (Tan et al., 2013; Xiong et al., 2014). Gold nanoparticles (AuNPs) are often used for aptasensor development, especially colorimetric detection schemes, due to their inherent advantages, such as easy preparation and good biocompatibility (Gopinath et al., 2014). Typically, the aptamer or aptamer-target binding on the gold nanoparticles induces their aggregation or dispersion (Soh et al., 2015; Chen et al., 2016). Crucially, gold nanoparticles with high extinction coefficient (Xie et al., 2012) and broad UV-Vis absorption (Andolina et al., 2013) are used as the quenchers for quantum dots through energy-transfer and electron-transfer process. Wang et al. devised aptamer-induced assembly of nitrogen-doped carbon dots on gold nanoparticles for fluorescent detection of aflatoxin $B_{1}$ (Wang et al., 2016b). Liu et al. demonstrated the surface energy transfer between carbon dots and gold nanoparticles (Liu et al., 2015). Mandani et al. found that ligand exchange with thiol containing biomolecules resulted in the release of carbon dots from the surface of gold nanoparticles leading to an enhancement of fluorescence (Mandani et al., 2015). To this end, our motivation in this work is expected to utilize terbium-coordinated carbon dots as the donors and gold nanoparticles as the acceptors for the development of fluorescent aptasensor.

Adenosine 5'-triphosphate (ATP) is often called the 'molecular unit of currency' of intracellular energy transfer. ATP serves as the mediator of energy exchanges in metabolic processes for daily activities, and also acts as an important character for biological determination to disease diagnosis 
(Zheng et al., 2015). Various methods and strategies have reported for the detection of ATP, e.g., by using fluorescent assay (Zhu et al., 2016), colorimetric detection (Huo et al., 2016), luminescence (Babu et al., 2016), electrochemiluminescent sensor (Zhao et al., 2016) and electrochemical technique ( $\mathrm{Lu}$ et al., 2015). Despite some advances in this field, there is still the request to explore some advanced detection schemes for improvement of sensitivity and feasibility. Herein we design a new time-resolved fluorescent aptasensing platform for sensitive detection of ATP coupling high-efficiency terbium-coordinated carbon dots (Tb-CDs) with gold nanoparticles (Scheme 1). The interaction of Tb-CDs with AuNPs is controlled by the newly formed aptamer-ATP complex in the presence of target ATP. The AuNPs are protected in high salt solution after formation of the aptamer-ATP complex, thus avoiding the aggregation of gold nanoparticles. The dispersive AuNPs efficiently quench the fluorescence of $\mathrm{Tb}$-CDs by fluorescence resonance energy transfer. The decreasing fluorescent signal depends on the concentration of target ATP. By monitoring the change in the fluorescence intensity, the level of target ATP can be evaluated. Further experimental processes are discussed in the following sections.

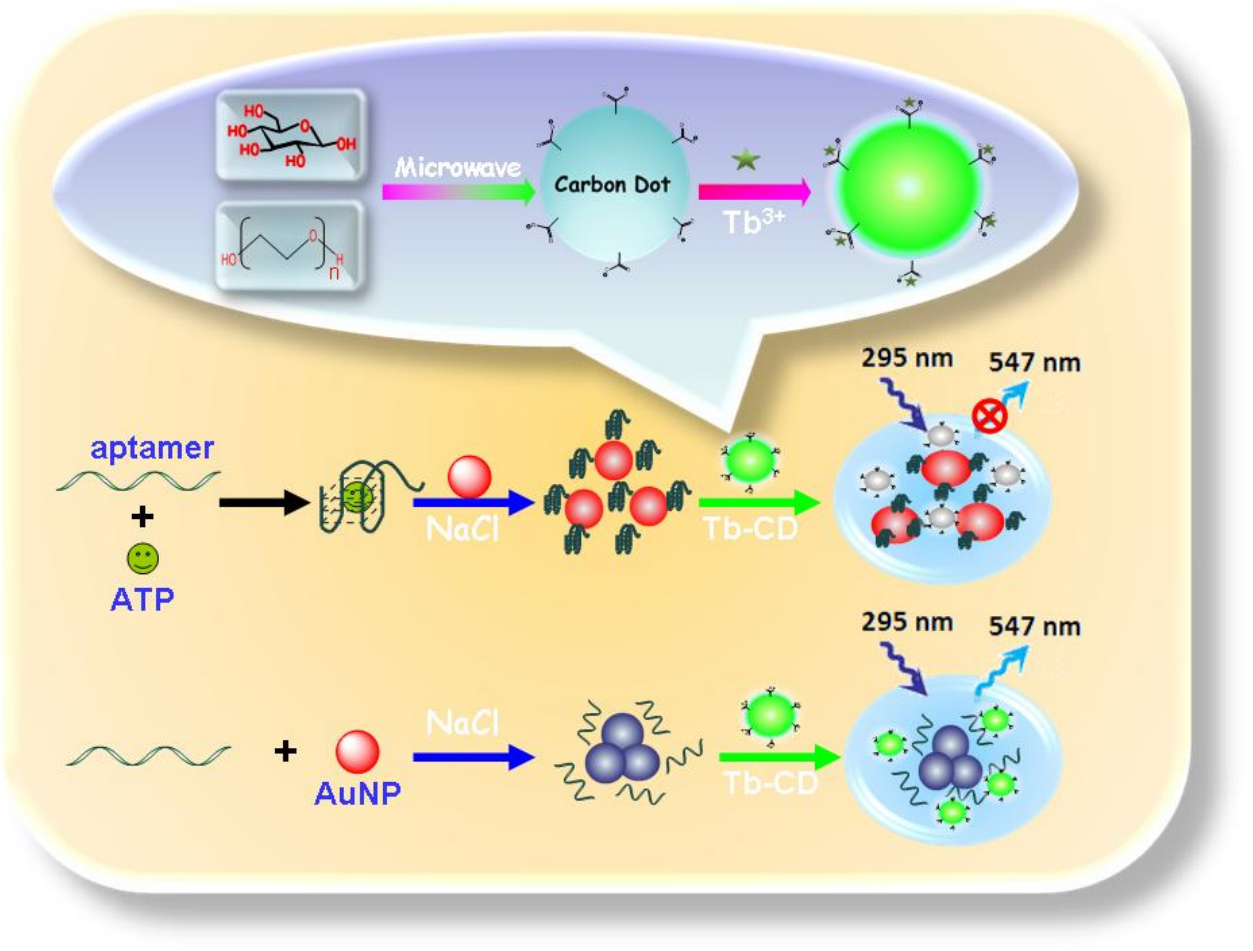

Scheme 1. Schematic illustration of fluorescent aptasensing platform for the quantitative monitoring of adenosine 5'-triphosphate (ATP) by interaction of unmodified gold nanoparticles (AuNPs) with terbium ion-functionalized carbon dots (Tb-CDs). 


\section{Experimental}

\subsection{Materials and apparatus}

Terbium chloride $\left(\mathrm{TbCl}_{3}\right)$ and PEG-200 were purchased from Dingguo Biotechnol. Co., Ltd. (Beijing, China). $\mathrm{HAuCl}_{4} \cdot 4 \mathrm{H}_{2} \mathrm{O}$, adenosine 5'-triphosphate (ATP), guanosine 5'-triphosphate (GTP), uridine 5'-triphosphate (UTP) and cytidine 5'-triphosphate (CTP) were achieved from SigmaAldrich (Shanghai, China). The chemicals of analytical grade were used throughout this study without further purification. Ultrapure water was used in all runs obtained from a Millipore water purification system (18.2 M $\mathrm{cm}^{-1}$, Milli-Q). $10 \mathrm{mM}$ Tris- $\mathrm{HCl}(\mathrm{pH}$ 7.4) was used for the binding buffer containing $20 \mathrm{mM} \mathrm{KCl}$. All the oligonucleotides were synthesized by Sangon Biotechnol. Inc. (Shanghai, China). The sequence of the ATP aptamer was as follows: 5'-ACC TGG GGG AGT ATT GCG GAG GAA GGT-3'.

Fluorescence spectra were measured on a Hitachi F-4600 fluorescence spectrofluorometer (Tokyo, Japan). The fluorescence lifetime was performed by using FLS920 (Edinburgh Instruments, Great Britain). The morphology of nanomaterials was characterized by high-resolution transmission electron microscopy (HRTEM, FEI F20, USA). UV-vis absorption spectra were obtained on a UV 1102 spectrophotometer (Techcomp, Shanghai, China). Fourier transform infrared spectra (FTIR) were recorded by a Nicolet iS50 (Thermo Fisher Nicolet, USA).

\subsection{Synthesis of carbon dots (CDs)}

Carbon dots (denoted as CDs) were synthesized using glucose as the carbon source and PEG-200 as the passivated particle on the basis of a domestic microwave method (Zhu et al., 2009). Initially, $2.0 \mathrm{~g}$ of glucose and $10 \mathrm{~mL}$ of $\left(10 \mathrm{mg} \mathrm{mL}^{-1}\right)$ PEG-200 were added into 3.0-mL ultrapure water, and the mixture was then vigorously stirred for $10 \mathrm{~min}$ at room temperature. Following that, the resulting suspension was put into a domestic microwave oven and heated for $5 \mathrm{~min}$ at $500 \mathrm{~W}$. During the pyrolysis process, the solution changed from colorless to dark brown accompanying the emanation of the smoke. Finally, the resultant product (i.e., carbon dots) was cooled to room temperature for further use.

\subsection{Preparation of terbium ion-coordinated carbon dots $(\mathrm{Tb}-\mathrm{CDs})$}

Terbium ion-coordinated carbon dots (denoted as $\mathrm{Tb}-\mathrm{CDs}$ ) were prepared similar to the literature 
with minor modification (Chen et al., 2015). Briefly, $5 \mathrm{~mL}$ of $100 \mathrm{mM} \mathrm{TbCl} \mathrm{T}_{3}$ aqueous solution was injected into $10 \mathrm{~mL}$ of $1.54 \mu \mathrm{g} \mathrm{mL}^{-1} \mathrm{CD}$ aqueous solution. Thereafter, the resulting mixture was vigorously stirred for $2 \mathrm{~h}$ at room temperature. During this process, terbium ions were assembled to carbon dots by the coordination chemistry with the carboxyl groups. After that, the resulting suspension was dialyzed for 3 days in cellulose ester dialysis membrane (1000 MWCO) to remove the redundant $\mathrm{Tb}^{3+}$ ions. Finally, the as-prepared $\mathrm{Tb}-\mathrm{CD}$ sere stored in the dark at room temperature.

\subsection{Preparation of gold nanoparticles (AuNPs)}

Gold nanoparticles (denoted as AuNPs) with $12 \mathrm{~nm}$ in diameter were prepared through typical citrate reduction method (Grabar et al., 1995). Initially, $10 \mathrm{~mL}$ of $38.8 \mathrm{mM}$ trisodium citrate was rapidly added into $100 \mathrm{~mL}$ of $1.0 \mathrm{mM}$ boiling $\mathrm{HAuCl}_{4}$ aqueous solution under vigorous stirring. Following that, the mixture was heated for 15 min under the same conditions. During this process, the solution changed from pale yellow to wine red. Finally, the as-synthesized gold colloids were cooled to room temperature and stored at $4{ }^{\circ} \mathrm{C}$ for use. The maximum absorbance of colloidal gold particles was examined at $520 \mathrm{~nm}$ in the UV-vis adsorption spectra and the extinction coefficient was $2.7 \times 10^{8} \mathrm{M}^{-1} \mathrm{~cm}^{-1}$. The size confirmed by TEM was approximate $12 \mathrm{~nm}$.

\subsection{Fluorescence measurement toward target ATP}

Scheme 1 represents the schematic illustration of time-resolved fluorescent aptasensing protocol toward target ATP. The assay was carried out as follows: (i) $5 \mu \mathrm{L}$ of ATP standards with different concentrations and $5 \mu \mathrm{L}$ of ATP aptamer $(250 \mathrm{nM})$ were added to $10 \mu \mathrm{L}$ of Tris-HCl buffer $(10 \mathrm{mM}$, $\mathrm{pH} 7.4,20 \mathrm{mM} \mathrm{KCl}$ ) and reacted for $15 \mathrm{~min}$ at room temperature; (ii) $100 \mu \mathrm{L}$ of 12-nm gold colloids $(17 \mathrm{nM})$ was injected to the mixture and equilibrated for 10 min under the same conditions; (iii) $10 \mu \mathrm{L}$ of $\mathrm{NaCl}$ aqueous solution $(500 \mathrm{mM})$ was added to the resulting suspension in order to induce the aggregation of the unprotected gold nanoparticles (Note: Until this step, the resultant suspension could be examined by UV-vis adsorption spectrometry); and (iv) $120 \mu \mathrm{L}$ of Tb-CDs (33 $\left.\mu \mathrm{M} \mathrm{mL}^{-1}\right)$ was added to the mixture for fluorescent measurement in a $1 \times 1 \mathrm{~cm}$ quartz cuvette $\left(\lambda_{\mathrm{ex}}=\right.$ $295 \mathrm{~nm}$ and $\lambda_{\mathrm{em}}=547 \mathrm{~nm}$ ). The change in the fluorescent intensity relative to background signal was collected and registered as the sensor signal relative to target ATP level. All measurements were 
performed at room temperature $\left(25 \pm 1.0^{\circ} \mathrm{C}\right)$. Analyses were always made in triplicate.

\section{Results and discussion}

\subsection{Design of AuNP-Tb-CD fluorescent aptasensing platform}

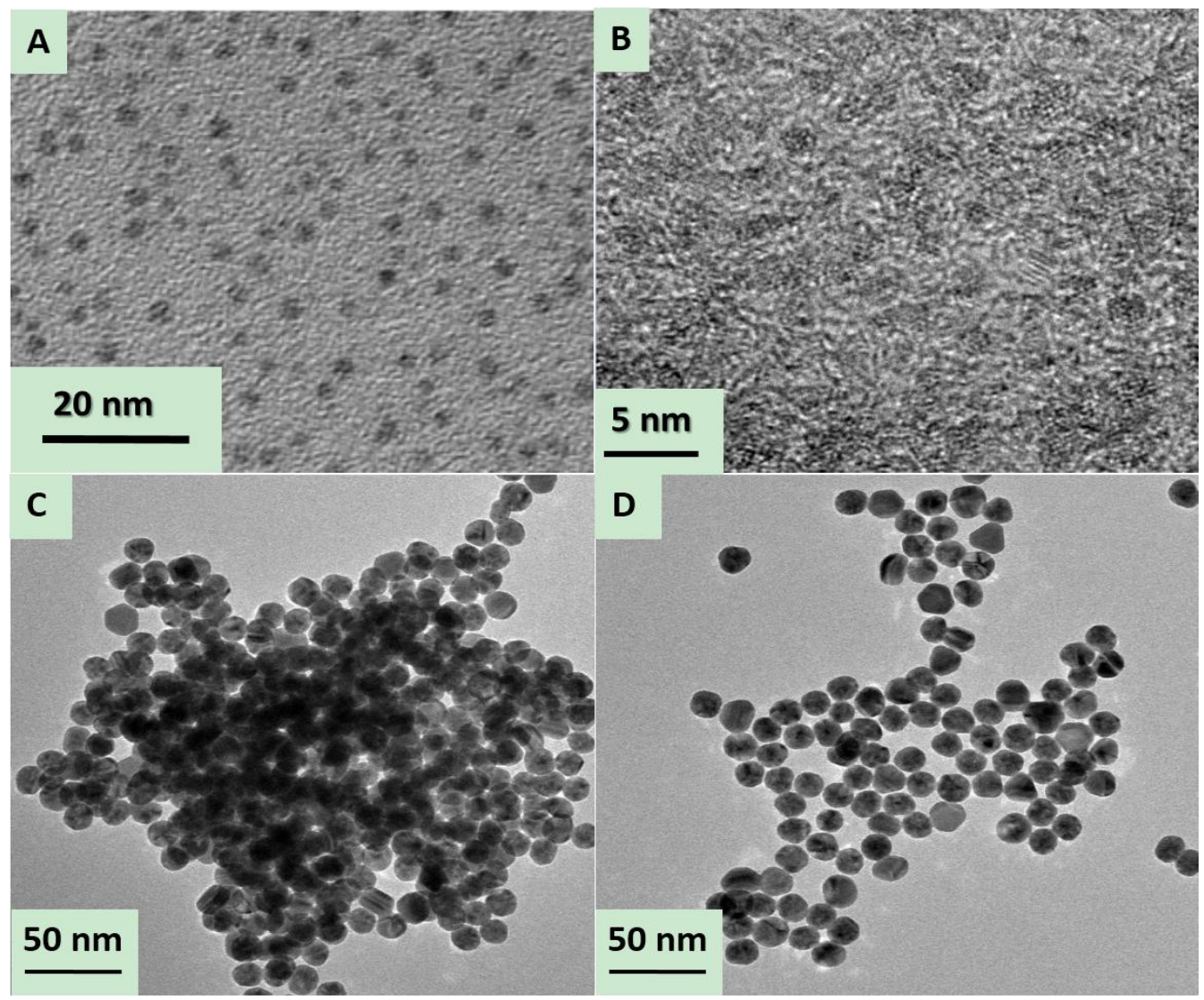

Fig. 1. TEM images of (A) Tb-CDs, (C) $13 \mathrm{nM}$ AuNPs $+125 \mathrm{nM}$ aptamer $+38 \mathrm{mM} \mathrm{NaCl}$ and (D) $13 \mathrm{nM}$ AuNPs $+125 \mathrm{nM}$ aptamer $+500 \mathrm{nM}$ ATP $+38 \mathrm{mM} \mathrm{NaCl}$ [Note: All the concentrations were the final concentrations), (B) HRTEM image of the as-synthesized Tb-CDs].

Typically, citrate-protected gold nanoparticles are stabilized in aqueous solution since the negatively charged citrate ions can prevent the van der Waals force from inducing their aggregation. Terbium ion-functionalized carbon dots with the strong fluorescent intensity are used as the nanotags for the determination of target ATP. Significantly, the fluorescent signal can be efficiently quenched by gold nanoparticles. Scheme 1 gives the detection principle of time-resolved fluorescent aptasensing mode. It was reported that single-stranded DNA could prevent gold nanoparticles for their aggregation in salt solution to some extent (Wang et al., 2007). However, the interaction was relatively weak. Recent work demonstrated that the formed aptamer-ATP complexes 
( similar to G-quadruplex structure) between the aptamer and target ATP could maintain the stability of gold nanoparticles more effectively than the aptamer alone in high salt solution (Huo et al., 2016). In the absence of target ATP, the added $\mathrm{NaCl}(500 \mathrm{mM})$ induces the aggregation of gold nanoparticles even if the aptamer is present in the solution. In this case, the Tb-CDs can efficiently remain their intrinsic fluorescent intensity. Upon target ATP introduction, the formed complexes between the analytes and the aptamers disperse around gold nanoparticles to avoid their aggregation in the salt solution. It may be explained that the aptamer-ATP complexes and the aptamer alone had different propensities for adsorption onto the AuNPs owing to their electrostatic properties, and the negative charges wee further extended from the nanogold surface in the presence of folded aptamer-ATP complexes than with unfolded aptamers (Huo et al., 2016). In this regards, the fluorescent signal of $\mathrm{Tb}-\mathrm{CDs}$ is quenched by the dispersive gold nanoparticles on the basis of fluorescence resonance energy transfer. Hence, the fluorescent signal can be determined through the interaction between Tb-CDs and AuNPs.

To realize our design, terbium-functionalized carbon dots were first synthesized via microwave and the coordination chemistry. Terbium ions were chelated with the carboxyl groups of carbon dots [Note: The carboxyl group was generated by oxidation of $\mathrm{C}-\mathrm{OH}$ bond from the decomposed oxygen atom under microwave condition (Zhu et al., 2009)]. Fig. 1A shows typical TEM image of the as-synthesized $\mathrm{Tb}-\mathrm{CDs}$, and the average size was $\sim 2.0 \mathrm{~nm}$ in diameter. Also, we observed that the as-prepared Tb-CDs could be homogeneously dispersed in ultrapure water. The high-solution transmission electron microscope (HRTEM) image in Fig. 1B exhibited a well-resolved lattice fringes. Further, the stretching vibrations of $\mathrm{C}=\mathrm{O}$ at $1610 \mathrm{~cm}^{-1}$ and $\mathrm{C}-\mathrm{O}-\mathrm{C}$ at $1300 \mathrm{~cm}^{-1}$ and 1200 $\mathrm{cm}^{-1}$ were obtained from fourier transform infrared (FTIR) spectroscopy. The results indicated the presence of carboxyl groups on the Tb-CDs (Zhu et al., 2009).

Another precondition for the development of fluorescent aptasensing platform relies on the fact whether the formed complex between the aptamer and target ATP could protect gold nanoparticles from avoiding their aggregation in salt solution. As shown from Fig. 1C, most gold nanoparticles were aggregated together in the absence of target ATP. The reason might be the fact that the added $\mathrm{NaCl}$ wrecked the interaction between the aptamer and nanogold particles. When target ATP was present in the system, favorably, numerous gold nanoparticles were dispersed in the solution (Fig. 1D). The results preliminarily revealed that gold nanoparticles readily dispersed in the presence of 
target ATP.

\subsection{Control tests of the AuNP-Tb-CD system}

To verify the feasibility of the AuNP-Tb-CD system, we used UV-vis adsorption spectroscope and fluorescence spectrometry to monitor their interaction between AuNP and Tb-CD. As shown from Fig. 2A, one obvious absorption peak was observed for pure terbium ions at $216 \mathrm{~nm}$ (curve ' $a$ '), gold nanoparticles at $520 \mathrm{~nm}($ curve ' $b$ ') and carbon dots at $279 \mathrm{~nm}$ (curve 'c') respectively. Significantly, two characteristic peaks at $218 \mathrm{~nm}$ and $280 \mathrm{~nm}$ could be simultaneously achieved at the as-prepared Tb-CDs (Fig. 2A, curve ' $d$ '). Relative to terbium ions and carbon dots alone, the shift in the peaks mainly derived from their interaction. A distinct surface plasmon band at $520 \mathrm{~nm}$ also indicated the successful synthesis of 12-nm gold colloids. As shown from curve ' $e$ ', upon the addition of Tb-CDs into gold colloids, a new adsorption peak at $650 \mathrm{~nm}$ was appeared, whilst the characteristic peak at $520 \mathrm{~nm}$ decreased, indicating the aggregation of the bigger-sized nanoparticles.
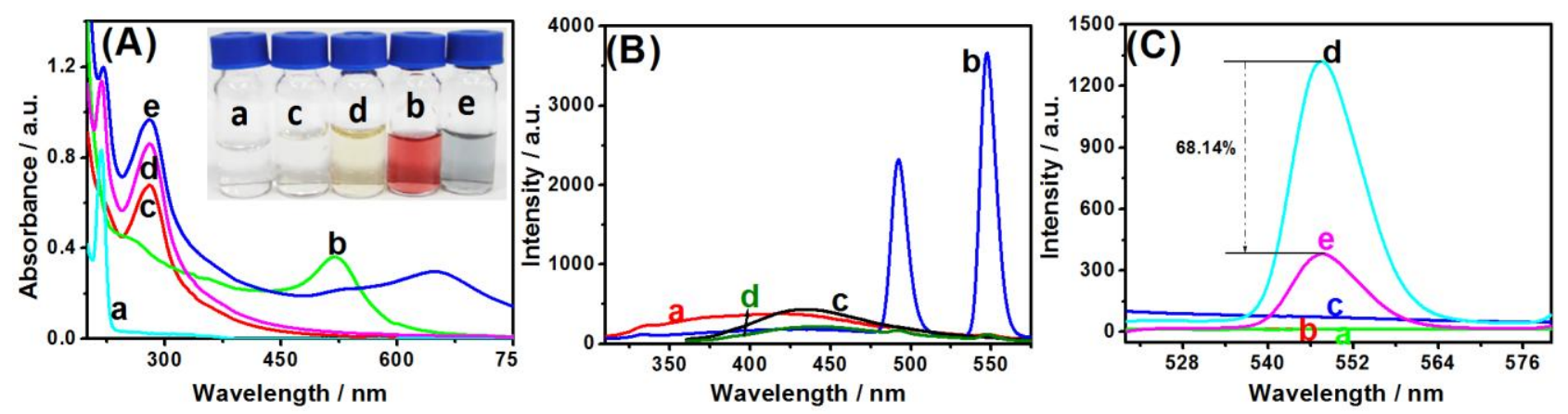

Fig. 2. (A) UV-vis absorption spectra and (C) fluorescence emission spectra at $\lambda_{\mathrm{ex}}=295 \mathrm{~nm}$ of (a) terbium ions, (b) AuNPs, $(c)$ carbon dots, $(d)$ Tb-CDs and (e) AuNPs + Tb-CDs (Insets: the corresponding photographs, but photograph ' $b$ ' was the diluted sample with the corresponding concentration of curve ' $b$ '). (B) Fluorescence emission spectra of $(a, c)$ carbon dots and $(b, d)$ Tb-CDs at $(a, b) \lambda_{\mathrm{ex}}=295 \mathrm{~nm}$ and $(c, d) \lambda_{\mathrm{ex}}=340 \mathrm{~nm}$.

In this work, carbon dots and terbium ions were used as the fluorescent probes. Naturally, one puzzling question to be produced was whether the fluorescent intensity of using the Tb-CDs was stronger than those of carbon dots or terbium ions alone. For comparison, we selected two emission wavelengths (295 nm and $340 \mathrm{~nm}$ ) (because $340 \mathrm{~nm}$ and $295 \mathrm{~nm}$ were suitable for the fluorescence emission of carbon dots and terbium ions, respectively) to investigate the fluorescence intensities of carbon dots and Tb-CDs under the same concentrations. As shown in Fig. 2B, the maximum 
fluorescence intensity was obtained for Tb-CDs at $\lambda_{\mathrm{ex}}=295 \mathrm{~nm}$. Therefore, use of Tb-CDs could amplify the fluorescence signal at $\lambda_{\mathrm{ex}}=295 \mathrm{~nm}$. Fig. $2 \mathrm{C}$ revealed the fluorescence behaviors of Tb-CDs centered at $547 \mathrm{~nm}$ when being excited at $295 \mathrm{~nm}$ (curve ' $d$ '). After the interaction between AuNPs and Tb-CDs, the fluorescence intensity decreased $\sim 68.14 \%$ of Tb-CDs alone (curve ' $e$ ') because of the quenching effect by AuNPs. As control tests, we also investigated the fluorescence properties of terbium ions, AuNPs and carbon dots. No fluorescence signals were acquired at this wavelength as indicated from curves ' $a-c$ ' in Fig. $2 \mathrm{C}$. These results further revealed that the as-synthesized $\mathrm{Tb}-\mathrm{CD}$ s could exhibit the strong fluorescence signal, which could be quenched by the added gold nanoparticles.

\subsection{Control experiments of the AuNP-ABA-Tb-CD system toward target ATP}

To explore a new research field with the above-mentioned AuNP-Tb-CD, this system was applied for detection of target ATP by coupling with the corresponding aptamer. Firstly, we used UV-vis absorption spectroscopy to monitor the characteristics of gold colloids in the absence and presence of target ATP and the aptamer, respectively. Relative to the citrate-protected AuNPs (Fig. 3A, curve ' $a^{\prime}$ ), the characteristic peak was shifted from $520 \mathrm{~nm}$ to $680 \mathrm{~nm}$ upon addition of $\mathrm{NaCl}$ (Fig. 3A, curve ' $e$ '). Moreover, the absorbance values of the AuNP-NaCl system at $680 \mathrm{~nm}$ were obviously different when ATP aptamer or ATP was present, respectively. As seen from curves 'c- $d$ ' in Fig. 3A, the presence of the aptamer or ATP in the AuNP-NaCl system also caused the increasing absorbance at $680 \mathrm{~nm}$, indicating that partial gold nanoparticles were aggregated together. Theoretically, we might be suspected that the simultaneous existence of the aptamer and ATP could induce the aggregation of more gold nanoparticles than those alone. However, a strange phenomenon was appeared as follows: The absorbance of the ABA-ATP-AuNP-NaCl system (at $680 \mathrm{~nm}$ ) (Fig. 3A, curve ' $b$ ') was less than those of the ABA-AuNP-NaCl (Fig. 3A, curve ' $c$ ') and the ATP-AuNP-NaCl system (Fig. 3A, curve ' $d$ '). The reason was ascribed to the fact that the added target ATP initially reacted with the aptamer to form a new ABA-ATP complex, which could protect gold nanoparticles from their aggregation in the high salt solution.

Logically, one question arises to whether AuNPs could also cause the change in the fluorescence signal of the as-synthesized Tb-CDs in the presence of the aptamer or target ATP. By the same token, the as-prepared AuNPs could strongly quench the fluorescence signal of Tb-CDs via fluorescence 
resonance energy transfer (Fig. 3B, curve ' $a$ '). When $\mathrm{NaCl}$ was added into the AuNP-Tb-CD system (Fig. 3B, curve ' $e$ '), however, the fluorescence intensity was close to that of pure Tb-CDs (Fig. 2C, curve ' $d$ '), indicating the aggregation of gold nanoparticles. In contrast, the fluorescence signals slightly decreased when the aptamer/or ATP alone was present in the AuNP-NaCl system (Fig. 3B, curves ' $c-d$ '), suggesting that the aptamer and target ATP alone could not inhibit the aggregation of gold nanoparticles in the $\mathrm{NaCl}$ solution. When the aptamer and target ATP were simultaneously existed in the system, significantly, the fluorescence signal decreased (Fig. 3B, curve ' $b$ '). These results were almost in accordance with those of UV-vis adsorption behaviors. So, our designed scheme was feasible for the detection of target ATP.
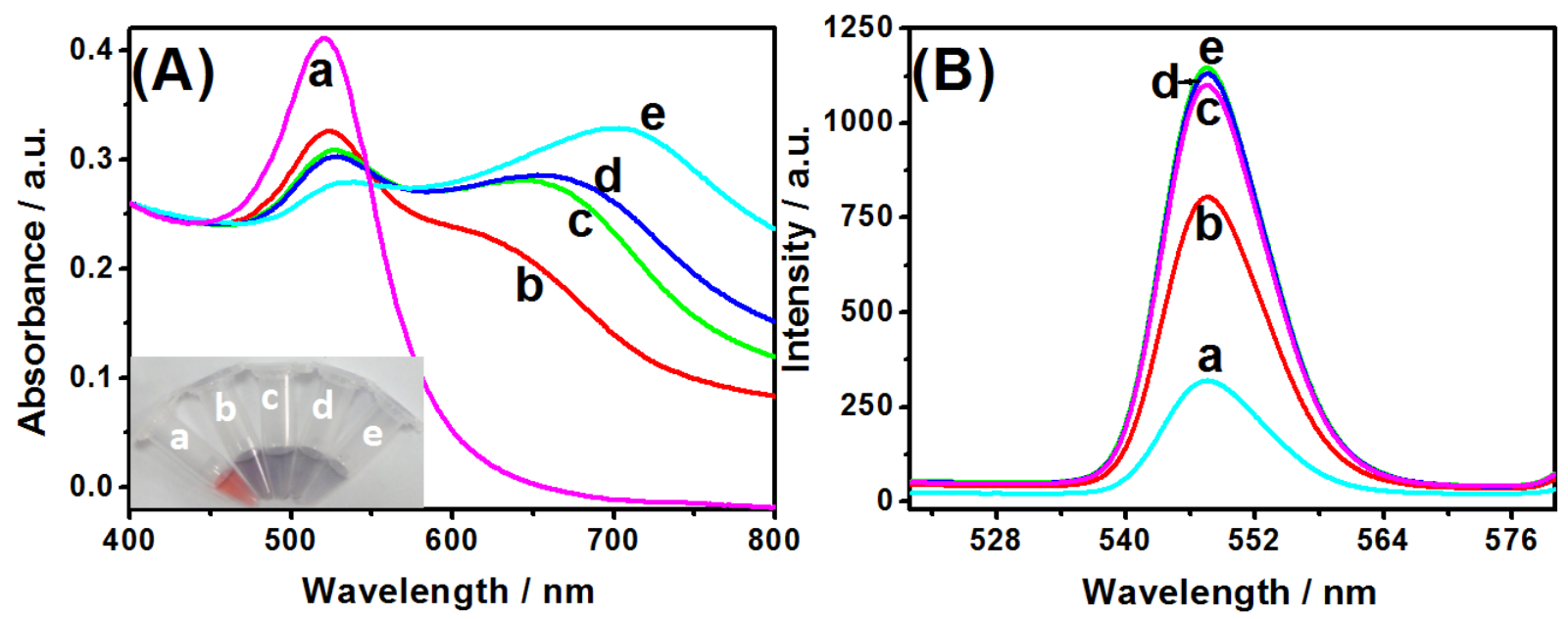

Fig. 3. (A) UV-vis absorption spectra of (a) $13 \mathrm{nM}$ AuNPs, (b) $125 \mathrm{nM}$ aptamer + $1.0 \mu \mathrm{M}$ ATP + $13 \mathrm{nM}$ AuNPs + $38 \mathrm{mM} \mathrm{NaCl},($ c) $125 \mathrm{nM}$ aptamer $+13 \mathrm{nM}$ AuNPs $+38 \mathrm{mM} \mathrm{NaCl},($ d) $1.0 \mu \mathrm{M}$ ATP + $13 \mathrm{nM}$ AuNPs $+38 \mathrm{mM}$ $\mathrm{NaCl}$ and (e) $13 \mathrm{nM}$ AuNPs $+38 \mathrm{mM} \mathrm{NaCl}$ (Insets: the corresponding photographs); (B) time-resolved fluorescence emission spectra of (a) $1.0 \mathrm{mM}$ AuNPs $+33 \mu \mathrm{M} \mathrm{mL}^{-1}$ Tb-CDs, (b) $250 \mathrm{nM}$ aptamer + $1.0 \mu \mathrm{M}$ ATP + $13 \mathrm{nM}$ AuNPs $+38 \mathrm{mM} \mathrm{NaCl}+33 \mu \mathrm{M} \mathrm{mL}^{-1} \mathrm{~Tb}-\mathrm{CDs}$, (c) $250 \mathrm{nM}$ aptamer $+13 \mathrm{nM} \mathrm{AuNPs}+38 \mathrm{mM} \mathrm{NaCl}+33$ $\mu \mathrm{M} \mathrm{mL}^{-1} \mathrm{~Tb}$-CDs, (d) $1.0 \mu \mathrm{M}$ ATP $+13 \mathrm{nM}$ AuNPs $+38 \mathrm{mM} \mathrm{NaCl}^{2}+33 \mu \mathrm{M} \mathrm{mL}^{-1} \mathrm{~Tb}-\mathrm{CDs}$ and $(e) 13 \mathrm{nM}$ AuNPs $+38 \mathrm{mM} \mathrm{NaCl}+33 \mu \mathrm{M} \mathrm{mL}^{-1} \mathrm{~Tb}-\mathrm{CDs}$ (Note: All the concentrations were the final concentrations).

\subsection{Dose responses of the fluorescence aptasensing platform toward target ATP}

Based on the fluorescence resonance energy transfer between AuNPs and Tb-CDs, the system was applied for quantitative monitoring of target ATP standards with different concentrations. Fig. 4A gives the time-resolved fluorescence spectra of the aptasensing platform. The emission intensity decreased with the increasing ATP concentration $\left(\lambda_{\mathrm{ex}}=295 \mathrm{~nm}\right.$ and $\left.\lambda_{\mathrm{em}}=547 \mathrm{~nm}\right)$. A good linear 
relationship between the fluorescence intensity $\left[\left(\mathrm{F}_{0}-\mathrm{F}\right) / \mathrm{F}_{0}\right]$ and ATP concentration could be obtained within the range from $40 \mathrm{nM}$ to $4.0 \mu \mathrm{M}$. The linear regression equation was fitted as $y=$ $0.1395 x+0.0875$ with a correlation coefficient of $0.996(n=7)$. The limit of detection (LOD) was evaluated to $8.5 \mathrm{nM}$ at $3 s_{\text {blank }}$ criterion, which was comparable with previously reported aptasensors, e.g., DNA/silver nanocluster-based fluorescence light-up system (91.6 nM) (Zhu et al., 2016), gold nanoparticles-based colorimetric assay (50 nM) (Huo et al., 2016), core-shell silver@ $\mathrm{SiO}_{2}$ nanoparticles-based fluorescent aptasensor (14.2 nM) (Song et al., 2016), AuNP-based fluorescence aptasensor (15.2 nM) (Meng et al., 2016) and Zinc(II)-protoporphyrin IX/G-quadruplex-based fluorescence aptasensor $(10 \mu \mathrm{M})$ (Zhang et al., 2012). To further elucidate the merit of using the $\mathrm{Tb}-\mathrm{CDs}$ for the development of fluorescence method, the AuNP-aptamer-NaCl system without Tb-CDs was also utilized for the detection of target ATP by using UV-vis absorption spectroscopy (Fig. 4B). The linear range and LOD were $0.2-6.0 \mu \mathrm{M}$ and $0.05 \mu \mathrm{M}$, respectively. Obviously, introduction of Tb-CDs could exhibit a low LOD and high sensitivity.

\subsection{Precision, reproducibility and selectivity}

To investigate the reproducibility of the fluorescent aptasensing platform, we repeatedly assayed three ATP concentrations including $0.04 \mu \mathrm{M}, 0.4 \mu \mathrm{M}$ and $2.0 \mu \mathrm{M}$, using identical batch Tb-CDs and AuNPs. Results revealed that the coefficients of variation (CVs) of the intra-assay were 5.4\%, 3.1\% and $6.2 \%$, while the CVs of the inter-assay with various batches were $7.9 \%, 9.4 \%$ and $8.3 \%$ toward the above-mentioned concentrations. Hence, the reproducibility of our system is satisfactory.

The specificity of the fluorescence aptasensing platform was evaluated by challenging our system against other biomolecules, and the results are listed in Fig. 4C-D. With an amount of non-target molecules, such as GTP, UTP and CTP, almost no apparent change in the signal was observed in comparison with that of the blank test in Fig. 4A-B. However, the presence of target ATP resulted in the dramatic shift in the signal relative to the blank test. The results indicated the high selectivity of our strategy. 

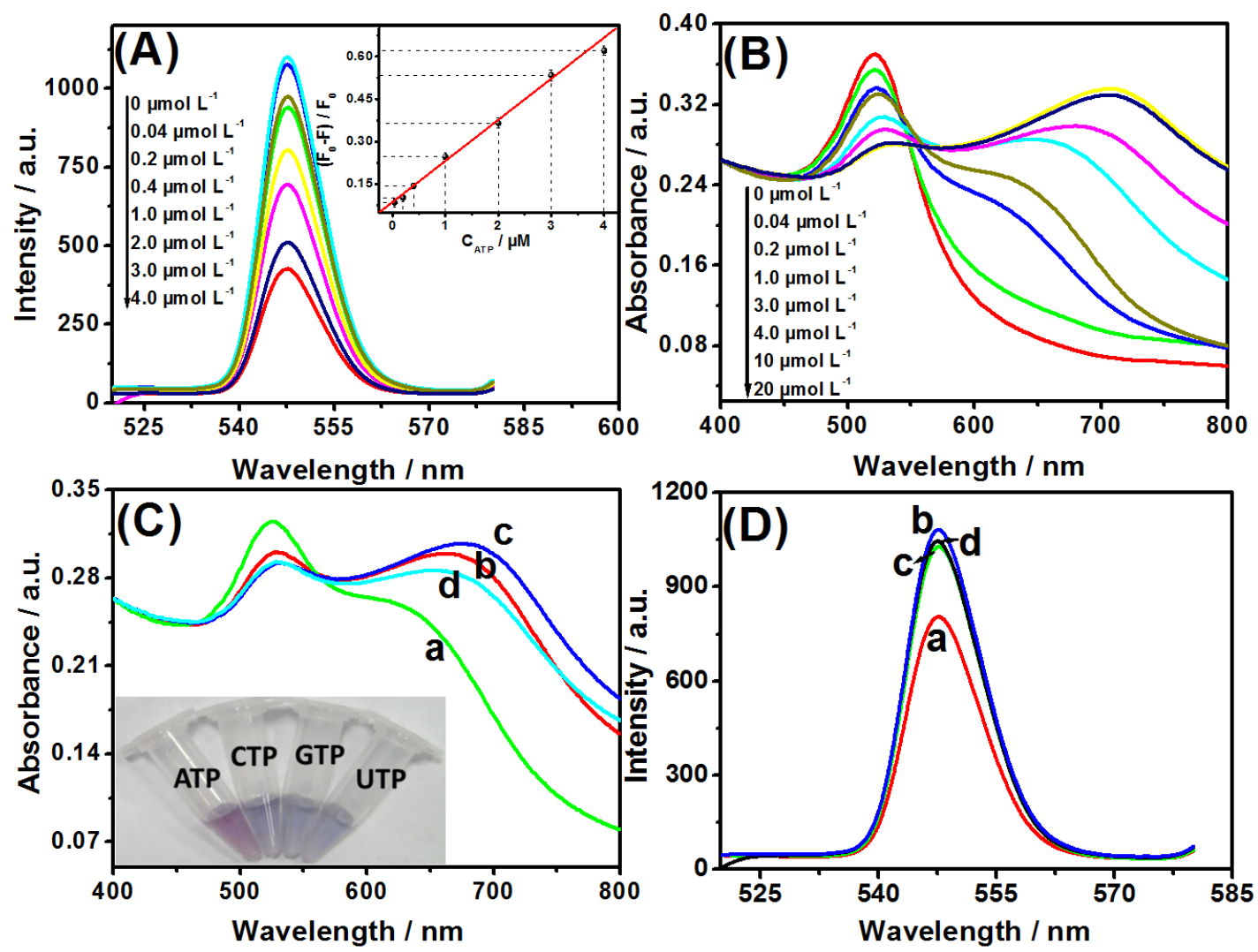

Fig. 4. (A) Time-resolved fluorescence emission spectra of the developed fluorescence aptasensing platform toward different-concentration ATP standards $\left(\lambda_{\mathrm{ex}}=295 \mathrm{~nm}\right.$ and $\left.\lambda_{\mathrm{em}}=547 \mathrm{~nm}\right)$ (Inset: the corresponding linear curve between $\left(\mathrm{F}_{0}-\mathrm{F}\right) / \mathrm{F}$ and ATP concentration), (B) UV-vis absorption spectra of the AuNP-aptamer-NaCl system toward ATP standards with various concentrations (Inset: the corresponding linear curve between A680/A520 and ATP concentration), and (C,D) the specificity of our system against (a) $1.0 \mu \mathrm{M}$ ATP, (b) $1.0 \mu \mathrm{M} \mathrm{CTP},(c) 1.0 \mu \mathrm{M}$ UTP and (d) $1.0 \mu \mathrm{M}$ GTP by using (C) UV-vis absorption spectroscopy and (D) fluorescence spectrometry (Inset: the corresponding photographs).

\subsection{Analysis of real sample}

To test the feasibility of the newly developed fluorescence aptasensing platform for analysis of target ATP in complex system, three ATP standards including $0.19 \mu \mathrm{M}, 0.96 \mu \mathrm{M}$ and $2.88 \mu \mathrm{M}$ were spiked into blank human serum samples. Initially, the blank human serum samples were 10-fold diluted with phosphate buffer ( $25 \mathrm{mM}$, containing $25 \mathrm{mM} \mathrm{NaCl}, \mathrm{pH} 7.0)$ prior to measurement (Yu et al., 2015), respectively. Then, the as-prepared samples were determined by using the fluorescence aptasensor at room temperature, and ATP concentrations were calculated according to our above-mentioned regression equation. The found levels of target ATP were 0.197 $\pm 0.036,0.947 \pm$ 0.011 and $2.792 \pm 0.039(n=3)$, respectively. The recoveries were $103.7 \%, 98.6 \%$ and $97.0 \%$ 
toward the above-mentioned analytes, respectively. Thus, our system could be preliminarily applied for the quantitative screening of target ATP in the complex sample.

\section{Conclusions}

In summary, we have successfully developed a facile and sensitive time-resolved fluorescence detection system for ATP relying on the highly quenching effect of gold nanoparticle to fluorescent Tb-CDs. Remarkably, the original fluorescent nanoprobes of Tb-CDs built by one-pot microwave synthesis afforded abundant carboxylic groups as the formworks to coordinate with terbium ions, which was able to send the distinct emission bands and largely amplify the individual signal of terbium ions. The fluorescence emission peak of Tb-CDs at $547 \mathrm{~nm}$ prominently distinguished from other substrates to eliminate unspecific fluorescent background signals (excited at $295 \mathrm{~nm}$ ). Moreover, our developed AuNP-Tb-CD energy transfer system exhibited particularly brilliant sensitivity, when the aptamer-ATP complex-induced protective AuNPs effectively quenched the characteristic fluorescence emission of $\mathrm{Tb}-\mathrm{CDs}$. Importantly, the remarkable flexibility and simplicity without any modification in this experimental design can be used as an alternative method for detecting other small biomolecules, thus paving the way for the development potential in biological and environmental applications.

\section{Acknowledgement}

Support by the National Natural Science Foundation of China (grant nos. $41176079 \& 21475025$ ), the National Science Foundation of Fujian Province (grant no. 2014J07001), and the Program for Changjiang Scholars and Innovative Research Team in University (grant no. IRT1116) is gratefully acknowledged. 


\section{References}

Amjadi, M., Manzoori, J., Hallaj, T., 2015. J. Lumin. 158, 160-164.

Andolina, C., Dewar, A., Smith, A., Marbella, L., Hartmann, M., Millstone, J., 2013. J. Am. Chem. Soc. 135, 5266-5269.

Babu, E., Mareeswaran, P., Ramdass, A., Ramesh, P., Rajagopal, S., 2016. J. Luminescence 175, 267-273.

Chen, B., Liu, Z., Zou, H., Huang, C., 2016. Analyst 141, 2676-2681.

Chen, H., Xie, Y., Kirillov, A., Liu, L., Yu, M., Liu, W., Tang, Y., 2015. Chem. Commun. 51, 5036-5039.

Chen, Z., Tan, L., Hu, L., Zhang, Y., Wang, S., Lv, F., 2016. ACS Appl. Mater. Interfaces 8, 102-108.

Crivian-Gaita, V., Thompson, M., 2016. Biosens. Bioelectron. 85, 32-45.

Dong, H., Kuzmanoski, A., Gossl, D., Popescu, R., Gerthsen, D., Feldmann, C., 2014. Chem. Commun. 50, 7503-7506.

Fong, J., Chin, S., Ng, S., 2016. Biosens. Bioelectron. 85, 844-852.

Gopinath, S., Lakshmipriya, T., Awazu, K., 2014. Biosens. Bioelectron. 51, 115-123.

Grabar, K., Freeman, R., Hommer, M., Natan, M., 1995. Anal. Chem. 67, 735-743.

Huo, Y., Qi, L., Lv, X., Lai, T., Zhang, J., Zhang, Z., 2016. Biosens. Bioelectron. 78, 315-320.

LeCroy, G., Yang, S., Yang, F., Liu, Y., Fernando, K., Bunder, C., Hu, Y., Luo, P., Sun, Y., 2016. Coordin. Chem. Rev. 320, 66-81.

Liu, S., Zhao, N., Chen, Z., Liu, H., 2015. Nanoscale 7, 6836-6842.

Lu, L., Si, J., Gao, Z., Zhang, Y., Lei, J., Luo, H., Li, N., 2015. Biosens. Bioelectron. 63, 14-20.

Mandani, S., Sharma, B., Dey, D., Sarma, T., 2015. Nanoscale 7, 1802-1808.

Meng, C., Dai, Z., Guo, W., Chu, Y., Chen, G., 2016. Nanomater. Nanotechnol. 6, art. No. 63985.

Prateek, V., Thakur, V., Gupta, R., 2016. Chem. Rev. 116, 4260-4317.

Sabherwal, P., Mutreja, R., Suri, C., 2016. TrAC Anal. Chem. 82, 12-21.

Schwenke, A., Hoeppener, S., Schubert, U., 2015. Adv. Mater. 27, 4113-4141.

Shi, B., Su, Y., Zhang, L., Huang, M., Liu, R., Zhao, S., 2016. ACS Appl. Mater. Interfaces 8, 10717-10725.

Soh, J., Lin, Y., Rana, S., Ying, J., Stevens, M., 2015. Anal. Chem. 87, 7644-7652.

Song, Q., Peng, M., Wang, L., He, D., Ouyang, J., 2016. Biosens. Bioelectron. 77, 237-241.

Stanisavljevic, M., Krizkova, S., Vaculovicova, M., Kizek, R., Adam, V., 2015. Biosens. Bioelectron. 74, 562-574.

Strehlitz, B., Reinemann, C., Linkom, S., Stoltenburg, R., 2012. Bioanal. Rev. 4, 1-30.

Tan, W., Donovan, M., Jiang, J., 2013. Chem. Rev. 113, 2842-2862.

Wang, N., Wang, Y., Guo, T., Yang, T., Chen, M., Wang, J., 2016a. Biosens. Bioelectron. 85, 68-75.

Wang, B., Chen, Y., Wu, Y., Weng, B., Liu, Y., Lu, Z., Li, C., Yu, C., 2016b. Biosens. Bioelectron. 78, 23-30.

Wang, J., Wang, L., Liu, X., Liang, Z., Song, S., Li, W., Fan, C., 2007. Adv. Mater. 19, 3943-3946 
Wen, J., Xu, Y., Li, H., Lu, A., Sun, S., 2015. Chem. Commun. 51, 11346-11358.

Wolfbeis, O., 2015. Chem. Soc. Rev. 44, 4743-4768.

Xie, X., Xu, W., Liu, X., 2012. Acc. Chem. Res. 45, 1511-1520.

Xiong, X., Lv, Y., Chen, T., Zhang, X., Wang, K., Tan, W., 2014. Ann. Rev. Anal. Chem. 7, 405-426.

Yu, P., He, X., Zhang, L., Mao, L., 2015. Anal. Chem. 87, 1373-1380.

Zhang, M., Yao, Q., Guan, W., Lu, C., Lin, J., 2014. J. Phys. Chem. C 118, 10441-10447.

Zhang, L., Wang, D., Huang, H., Liu, L., Zhou, Y., Xia, X., Deng, D., Liu, X., 2016. ACS Appl. Mater. Interfaces $8,6646-6655$.

Zhang, L., Diao, S., Nie, Y., Yan, K., Liu, N., Bai, Y., Xie, Q., Reina, A., Liu, Z., 2011a. J. Am. Chem. Soc. 133, 2706-2713.

Zhang, Y., Tang, Z., Fu, X., Xu, Y., 2011b. ACS Nano 5, 7426-7435.

Zhang, Z., Sharon, E., Freeman, R., Liu, X., Willner, I., 2012. Anal. Chem. 84, 4789-4797.

Zhao, T., Lin, C., Yao, Q., Chen, X., 2016. Talanta 154, 492-497.

Zheng, F., Zhang, P., Xi, Y., Chen, J., Li, L., Zhu, J., 2015. Anal. Chem. 87, 11739-11745.

Zhu, H., Wang, X., Li, Y., Wang, Z., Yang, F., Yang, X., 2009. Chem. Commun. 45, 5118-5120.

Zhu, Y., Hu, X., Shi, S., Gao, R., Huang, H., Zhu, Y., Lv, X., Yao, T., 2016. Biosens. Bioelectron. 79, 205-212. 